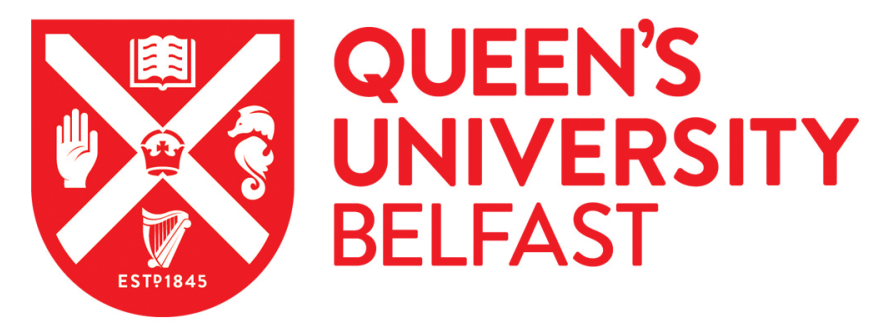

\title{
Generation of overdense and high-energy electron-positron-pair plasmas by irradiation of a thin foil with two ultraintense lasers
}

Chang, H. X., Qiao, B., Xu, Z., Xu, X. R., Zhou, C. T., Yan, X. Q., Wu, S. Z., Borghesi, M., Zepf, M., \& He, X. T. (2015). Generation of overdense and high-energy electron-positron-pair plasmas by irradiation of a thin foil with two ultraintense lasers. Physical Review E, 92(5), [053107 ]. https://doi.org/10.1103/PhysRevE.92.053107

Published in:

Physical Review E

Document Version:

Publisher's PDF, also known as Version of record

Queen's University Belfast - Research Portal:

Link to publication record in Queen's University Belfast Research Portal

Publisher rights

C2015 American Physical Society

\section{General rights}

Copyright for the publications made accessible via the Queen's University Belfast Research Portal is retained by the author(s) and / or other copyright owners and it is a condition of accessing these publications that users recognise and abide by the legal requirements associated with these rights.

Take down policy

The Research Portal is Queen's institutional repository that provides access to Queen's research output. Every effort has been made to ensure that content in the Research Portal does not infringe any person's rights, or applicable UK laws. If you discover content in the Research Portal that you believe breaches copyright or violates any law, please contact openaccess@qub.ac.uk. 


\title{
Generation of overdense and high-energy electron-positron-pair plasmas by irradiation of a thin foil with two ultraintense lasers
}

\author{
H. X. Chang, ${ }^{1,2}$ B. Qiao, ${ }^{1,2, *}$ Z. Xu, ${ }^{1,2}$ X. R. Xu, ${ }^{1,2}$ C. T. Zhou ${ }^{1,3}$ X. Q. Yan, ${ }^{1}$ S. Z. Wu, ${ }^{3}$ \\ M. Borghesi, ${ }^{4}$ M. Zepf,${ }^{4}$ and X. T. $\mathrm{He}^{1,3}$ \\ ${ }^{1}$ Center for Applied Physics and Technology, HEDPS, State Key Laboratory of Nuclear Physics and Technology, \\ and School of Physics, Peking University, Beijing 100871, China \\ ${ }^{2}$ Collaborative Innovation Center of Extreme Optics, Shanxi University, Taiyuan, Shanxi 030006, China \\ ${ }^{3}$ Institute of Applied Physics and Computational Mathematics, Beijing 100094, China \\ ${ }^{4}$ Department of Physics and Astronomy, Queen's University Belfast, Belfast BT7 1NN, United Kingdom
}

(Received 7 May 2015; published 17 November 2015)

\begin{abstract}
A scheme for enhanced quantum electrodynamics (QED) production of electron-positron-pair plasmas is proposed that uses two ultraintense lasers irradiating a thin solid foil from opposite sides. In the scheme, under a proper matching condition, in addition to the skin-depth emission of $\gamma$-ray photons and Breit-Wheeler creation of pairs on each side of the foil, a large number of high-energy electrons and photons from one side can propagate through it and interact with the laser on the other side, leading to much enhanced $\gamma$-ray emission and pair production. More importantly, the created pairs can be collected later and confined to the center by opposite laser radiation pressures when the foil becomes transparent, resulting in the formation of unprecedentedly overdense and high-energy pair plasmas. Two-dimensional QED particle-in-cell simulations show that electron-positron-pair plasmas with overcritical density $10^{22} \mathrm{~cm}^{-3}$ and a high energy of $100 \mathrm{~s}$ of $\mathrm{MeV}$ are obtained with $10 \mathrm{PW}$ lasers at intensities $10^{23} \mathrm{~W} / \mathrm{cm}^{2}$, which are of key significance for laboratory astrophysics studies.
\end{abstract}

DOI: 10.1103/PhysRevE.92.053107

Ever since Dirac [1] first postulated the existence of antimatter and Anderson [2] first identified positrons in cloud chambers, the creation of electron-positron pair plasma in the laboratory has been a subject of paramount importance because such plasma represents a unique state of matter consisting of negatively charged (matter) and positively charged (antimatter) particles in intrinsic and complete symmetry. Electron-positron pair production was first achieved in 1997 at the Stanford Linear Accelerator Center (SLAC) [3], where only $106 \pm 14$ positrons were observed. Positrons and positron plasmas from such large conventional accelerators or radioactive isotopes have been studied extensively at low energies (sub-MeV) in areas related to surface science, positron emission tomography, basic antimatter science (such as antihydrogen experiments) [4], and Bose-Einstein condensed positronium $[5,6]$.

Alternatively, dense relativistic $(>\mathrm{MeV})$ pair plasmas play a fundamental role in the dynamics of many astrophysical objects, such as violent emission of $\gamma$-ray bursts [7,8], black holes [9,10], and acceleration of cosmic rays [11]. A series of studies on these phenomena can be achieved using ad hoc laboratory experiments. However, the extreme difficulty in the current laboratory astrophysics studies is determining how to generate electron-positron plasmas that are dense enough to permit collective and kinetic behaviors to play roles, so that they are similar to the condition of astrophysical events such as jets of long $\gamma$-ray bursts $[7,8]$. Furthermore, to open up the possibility of studying the dynamics of astrophysical pair plasma, the created plasma needs to have a high energy, such as that with a Lorentz factor $\gamma \geqslant 15$ with a power-law

\footnotetext{
*Author to whom all correspondence should be addressed: bqiao@pku.edu.cn
}

PACS number(s): 52.38.Ph, 52.27.Ep, 79.20.Mb

spectral distribution comparable to what was observed in the astrophysical jets [12].

The use of intense lasers represents a promising approach to create such dense relativistic pair plasmas. One method involves using intense lasers to irradiate a thick high- $Z$ target, where a large amount of accelerated fast electrons radiates $\mathrm{MeV}$ bremsstrahlung photons in the target, which, in turn, produce pairs through the Bethe-Heitler process [13-15]. Recent experiments [16-18] have measured up to $2 \times 10^{10}$ positrons per steradian at a low density of $10^{16} \mathrm{~cm}^{-3}$ with an effective temperature of $2-4 \mathrm{MeV}$ using this method.

On the other hand, because of the impressive progress in laser technology, laser intensities of $2 \times 10^{22} \mathrm{~W} / \mathrm{cm}^{2}$ [19] are now available and are expected to reach $10^{23}-10^{24} \mathrm{~W} / \mathrm{cm}^{2}$ [20] in the next few years. For plasma interaction with such ultraintense lasers, the ratio of the electromagnetic fields in the electron's rest frame to the Schwinger field [21] $\left(E_{s}=m^{2} c^{3} / e \hbar=1.3 \times 10^{18} \mathrm{~V} \mathrm{~m}^{-1}\right)$ can exceed unity, i.e., $\eta=\left(e \hbar / m_{e}^{3} c^{4}\right)\left|F_{\mu \nu} p^{\nu}\right| \approx\left(\gamma / E_{s}\right)\left|\mathbf{E}_{\perp}+\beta \times c \mathbf{B}\right| \gtrsim 1$, where $\gamma$ is the Lorentz factor of a fast electron, $m$ is the electron mass, $e$ is the electron charge, $c$ is the light speed, $\beta$ is the velocity normalized to $c$, and $\mathbf{E}_{\perp}$ is the electric field perpendicular to its motion direction. Therefore, nonlinear quantum electrodynamics (QED) [22] reactions start to dominate in this regime. A significant number of electron-positron pairs can be produced through the multiphoton Breit-Wheeler process [23], $\gamma+n \gamma_{l} \rightarrow e^{+}+e^{-}$, where $\gamma_{l}$ is a laser photon, and $e^{+}$and $e^{-}$indicate the created positron and electron, respectively. $\gamma$ represents the emitted $\gamma$-ray photons through nonlinear Compton scattering from relativistic electrons in the laser fields, i.e., $e+n \gamma_{l} \rightarrow e^{\prime}+\gamma$. During the interaction, electrons emit photons, photons emit pairs, and particles (electrons, photons, and positrons) suffuse the interaction area, achieving cascade pair production. A general configuration of cascade pair production uses irradiation of very-low-density 
(transparent) targets with two counterpropagating lasers, where the targets just play the role of a seed with no collective and kinetic effects. Theoretically, the necessary condition for the occurrence of such cascade pair production requires the laser intensity $I>2.5 \times 10^{25} \mathrm{~W} / \mathrm{cm}^{2}$ [22]. Particle-in-cell (PIC) Monte Carlo (MC) simulations [24-27] have shown that this can be achieved with $100 \mathrm{PW}$ lasers at intensities of $3 \times 10^{24} \mathrm{~W} / \mathrm{cm}^{2}$. Recent QED-PIC simulations [28-30] have shown that such a pair plasma can be generated with an order of magnitude less laser power by firing the laser at a solid target, putting such experiments in reach of the next generation of 10 petawatt (PW) lasers $\left(I=4 \times 10^{23} \mathrm{~W} / \mathrm{cm}^{2}\right)$, where the plasma density can rise up to $10^{20} \mathrm{~cm}^{-3}$ while still being underdense.

In this paper, we propose a QED scheme for enhanced production of overdense and high-energy electron-positron pair plasmas, where two ultraintense laser pulses are used to irradiate a thin solid foil target from opposite sides. By satisfying a proper matching condition between laser and foil parameters, the high-energy electrons, the emitted $\gamma$-ray photons, and the created electron-positron pairs can all adequately interact with each other and with the laser fields at both sides of the foil with almost no leaks from the interaction area. More importantly, when the foil starts thermal expansion and becomes transparent at a later time, the created pairs are finally collected into the center by the incoming lasers and trapped [31,32] there by the standing waves formed directly by two laser pulses. Due to the strongly coupled collective, kinetic, and QED effects, our scheme eventually results in much enhanced positron production and the formation of unprecedentedly dense and high-energy pair plasmas. Two-dimensional (2D) QED-PIC simulations have verified the idea and shown that high-energy (100s of MeV up to $\mathrm{GeV}$ ) overdense electron-positron pair plasmas with density up to $4.5 \times 10^{22} \mathrm{~cm}^{-3}$ (particle number $1.2 \times 10^{12}$ ), two orders of magnitude higher than obtained previously [28-30], are obtained by irradiation of an $\mathrm{Al}$ solid foil with $10 \mathrm{PW}$ lasers at intensities $3.4 \times 10^{23} \mathrm{~W} / \mathrm{cm}^{2}$.

The enhanced pair production scheme uses two laser pulses to irradiate on a thin solid foil target from opposite sides. The enhancement of $\gamma$-ray emission and pair production comes from four aspects. First, at the initial "hole-boring" stage [33-36], the foil electrons and ions are accelerated and compressed by intense lasers from both sides. Due to the opposite, equal laser radiation pressures, high-energy electrons can be well confined in the foil without any leaks. Therefore, the foil is compressed to much higher density with higher reflectivity of the laser, and more stable standing waves are formed on each side of the foil. The larger reflected laser increases the skin-depth emission [37] of $\gamma$-ray photons. More stable standing waves help the emitted photons interact more adequately with the laser fields, leading to much enhanced pair production through the Breit-Wheerler process [28]. In addition, they help to trap the created pairs at the nodes $(E=0)$ of the standing waves, avoiding their dispersal. Secondly, in addition to the $\gamma$-ray emission and corresponding pair production on each side of the foil, a large number of accelerated high-energy electrons from one side of the foil can rapidly propagate through it and interact with the laser on the other side, resulting in significantly increased $\gamma$-ray emission and consequently enhanced pair creation. Thirdly, similarly, the high-energy $\gamma$-ray photons produced on one side of the target can also propagate through the foil (with almost no loss due to their high frequency) and interact with the laser on the other side, creating additional large numbers of pairs via the Breit-Wheeler process as well. Lastly, but most importantly, when the hole boring ends, the foil starts thermal expansion (the sum of the electrostatic and thermal pressures exceeds the laser radiation pressures) until the laser eventually punches through. Afterward, the created pairs are accelerated and finally collected to the center and retrapped by the new standing waves formed directly by the two lasers, which results in the production of extremely dense and high-energy electron-positron pair plasmas.

In the above scheme, the laser hole-boring velocity $v_{b}$ can be estimated as $v_{b} / c=\sqrt{\Xi} /(1+\sqrt{\Xi})$ [35], where $\Xi=$ $I_{0} / m_{i} n_{i} c^{3}=I / \rho c^{3}$ is the dimensionless pistoning parameter, $\rho=m_{i} n_{i}$ is the foil mass density, and $m_{i}$ and $n_{i}$ are the ion mass and the density. With an increase of the mass density due to continuous piling up, $v_{b}$ drops significantly to almost zero when the hole boring ends, where the radiation pressure is balanced by the electrostatic plus the thermal pressures of the compressed foil. The time duration of this hole-boring stage can be estimated as

$$
\tau_{\mathrm{hb}} \approx \frac{l_{0}}{2 v_{b}}=\frac{l_{0}}{2 c} \frac{1+\sqrt{\Xi}}{\sqrt{\Xi}},
$$

where $l_{0}$ is the foil thickness. Later, when the left compression layer of the foil overlaps with the right one, both the electrostatic and thermal pressures increase rapidly and their sum exceeds the laser radiation pressure on each side, leading to heavy decompression and expansion of the foil. Eventually, the foil becomes transparent to the laser. Assuming the thermal expansion dominates here, due to the conservation of the total particle number in the foil as $n_{0} l_{0}=n_{s} l_{s} \approx \gamma n_{c}\left(2 c_{s} \tau_{\mathrm{ep}}+\right.$ $l_{s}$ ) [38], where $n_{s}$ is the maximum density that the foil achieves after compression and $l_{s}$ is the corresponding thickness, this expansion duration can be roughly estimated as

$$
\tau_{\mathrm{ep}} \approx \frac{n_{0} l_{0}}{2 \gamma n_{c} c_{s}},
$$

where $c_{s}=\sqrt{T_{e} / m_{i}}$ is the ion sound speed and $n_{c}=$ $m \epsilon_{0} \omega^{2} / e^{2}$ is the critical density. To achieve the whole process illustrated above, the laser pulse duration $\tau_{L}$ must be larger than the sum of $\tau_{\mathrm{hb}}$ and $\tau_{\mathrm{ep}}$, i.e.,

$$
\tau_{L}>\tau_{\mathrm{hb}}+\tau_{\mathrm{ep}}
$$

Furthermore, if all the foil electrons are blown out by the laser radiation pressure initially, no hole-boring stage exists. Thus, the laser radiation pressure should be smaller than the maximum electrostatic pressure, i.e.,

$$
I_{0} / c<2 \pi n_{0}^{2} e^{2} l_{0}^{2} .
$$

Equations (1)-(4) give the matching condition of laser and target parameters to achieve our scheme.

To verify our scheme, 2D PIC simulations are carried out using the QED-PIC code EPOCH [37], which takes into account the important QED effects [39] in the synchrotron radiation of $\gamma$-rays and Breit-Wheeler production of electron-positron 

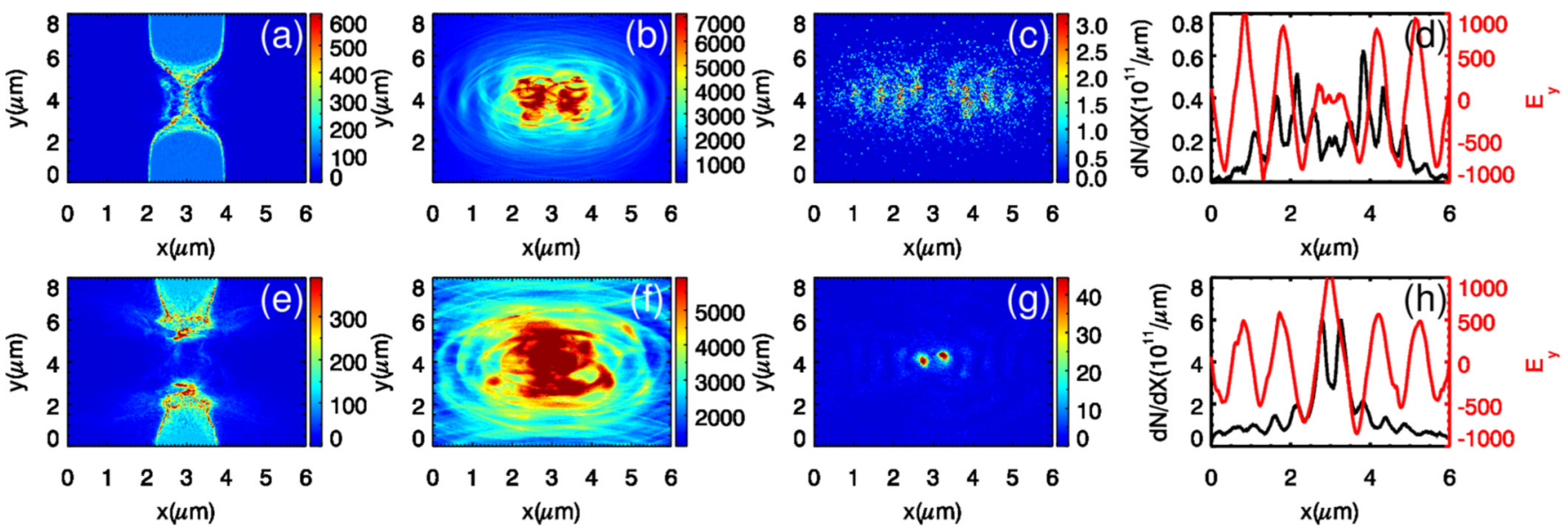

FIG. 1. (Color online) The density maps in units of $n_{c}$ of the $\mathrm{Al}^{13+}$ ions [(a) and (e)], emitted $\gamma$-ray photons [(b) and (f)], and created positrons [(c) and (g)] at $t=9 T_{0}$ (upper row) and $18 T_{0}$ (bottom row), respectively, where two ultraintense lasers at the same intensities $I_{0}=3.4 \times 10^{23} \mathrm{~W} / \mathrm{cm}^{2}$ irradiate a $2 \mu \mathrm{m} \mathrm{Al}$ foil target from opposite sides. Parts (d) and (h) are the corresponding longitudinal distributions of positron numbers (black) and the longitudinal profiles of the normalized electric fields $E_{y}$ (red).

pairs by a Monte Carlo algorithm [40]. In the simulations, 600 cells along the $x$ axis and 800 cells transversely along the $y$ axis constitute a $6 \times 8 \mu \mathrm{m}$ simulation box. A fully ionized aluminum (Al) foil with solid density $\rho_{0}=2.7 \mathrm{~g} / \mathrm{cm}^{3}$ $\left(\mathrm{Al}^{13+}\right.$ density $n_{i}=6 \times 10^{22} \mathrm{~cm}^{-3}$ ) and thickness $l_{0}=2 \mu \mathrm{m}$ is located at $x=2 \mu \mathrm{m}$. Each foil cell is filled up with 500 pseudoelectrons and 64 ions. Two linearly $y$-polarized lasers with the same intensities $I_{0}=3.4 \times 10^{23} \mathrm{~W} / \mathrm{cm}^{2}$ (normalized amplitudes $a_{0}=500$ ) and wavelengths $\lambda=1 \mu \mathrm{m}$ propagate from, respectively, the left and right boundaries and are incident on opposite sides of the foil. The laser pulses have transverse Gaussian profiles of full-width-at-half-maximum (FWHM) radius $r_{0}=2 \mu \mathrm{m}$ and a square temporal profile of durations $\tau=60$ fs $\left(18 T_{0}, T_{0}=2 \pi / \omega_{0}\right)$. The laser and target conditions satisfy the above conditions (3) and (4) of our enhanced scheme. Note that the hot electrons produced in such short-pulse laser-plasma interactions can have 100s of $\mathrm{MeV}$ energies, whose typical mean free path lengths in the moderate $Z$ foil target reach about 100 s of millimeters, much larger than the foil thickness, therefore the Bethe-Heitler process $[16,17,41]$ is rather weak and can be neglected here.

Figure 1 shows density maps of $\mathrm{Al}^{13+}$ ions [(a) and (e)], $\gamma$-ray photons [(b) and (f)], and created positrons [(c) and (g)] at times $t=9 T_{0}$ and $18 T_{0}$, respectively. At the initial holeboring stage [Fig. 1(a)], consistent with the above theoretical expectation, the foil is highly compressed from both sides by two laser radiation pressures. According to Eq. (1), the average hole-boring velocity $v_{b}$ is about $5.0 \times 10^{7} \mathrm{~m} / \mathrm{s}$, which ends at about $t=9 T_{0}$ when the laser radiation pressure is balanced by the electrostatic pressure plus the thermal pressure of the compressed layer. As shown in Fig. 2(f) at $t=9 T_{0}$, the maximum $\mathrm{Al}^{13+}$ density (laser-faced surface) increases from an initial $60 n_{c}$ up to $600 n_{c}$. Also as predicted by the theory, higher density and larger reflectivity of the foil lead to the formation of a more stable standing wave [Fig. 2(c)] on each side, in comparison with that in the case of using one laser pulse [Fig. 2(a)], whose antinodes are located at $x=0.2,0.7, \ldots, 2.7 \mu \mathrm{m}$ on the left side and symmetrically distributed on the other side [this is shown in Fig. 2(c) as well]. The foil electrons are accelerated to extremely high energy up to $\mathrm{GeV}$, as seen in Fig. 2(e), and they can easily achieve synchrotron emission of $\gamma$-ray photons.
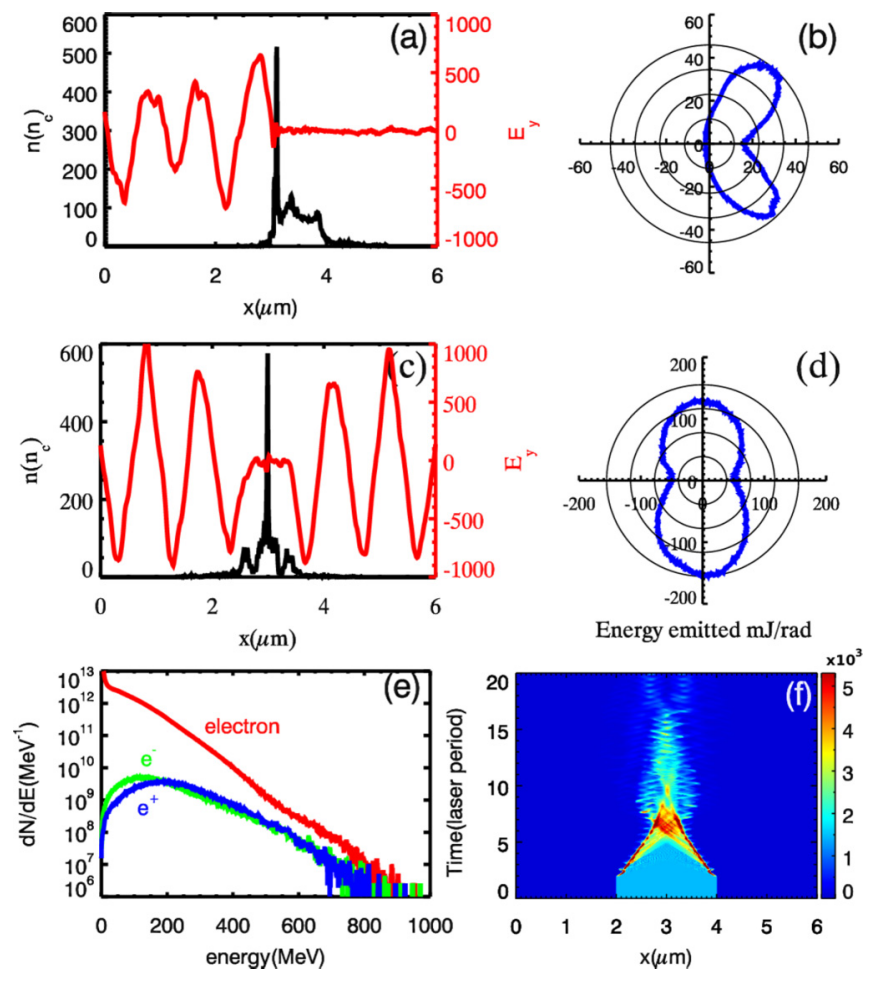

FIG. 2. (Color online) The longitudinal profiles of a laser electric field $E_{y}$ (red) and $\mathrm{Al}^{13+}$ density $n_{i} / n_{c}$ (black) at $y=0$ and $T=9 T_{0}$ for the cases of a $2 \mu \mathrm{m} \mathrm{Al}$ foil irradiated by one laser pulse (a) and by two laser pulses (c), respectively, where the laser and target parameters are the same as in Fig. 1. Parts (b) and (d) are, respectively, the angular distributions of the emitted $\gamma$-ray photon energies for two cases. Part (e) is the corresponding energy spectra of foil electrons, pair electrons, and positrons at $t=18 T_{0}$ in Fig. 1. Part (f) shows the evolution with time of the corresponding foil electron density longitudinal profile. 
The synchrotron emission of $\gamma$-ray photons due to interaction of high-energy electrons with lasers is shown in Fig. 1(b) at $t=9 T_{0}$, whose energy angular distribution is shown in Fig. 2(d). On the one hand, we can see that both the total emitted $\gamma$-ray photon number and the energy in our scheme are much larger than those in the one-laser case [Fig. 2(b)]. The total photon number at $t=9 T_{0}$ is about $6.65 \times 10^{13}$, which is 2.5 times larger than that in the one-laser case, whose average energy is about $5 \mathrm{MeV}$ with a maximum value of $520 \mathrm{MeV}$. This increase of $\gamma$-ray photon emission is due to the interaction of the laser on one side of the foil with the ponderomotively accelerated (or $\mathbf{j} \times \mathbf{B}$ heated) high-energy electrons transported and generated from the other side, in addition to the known skin depth emission on only one side. On the other hand, it shows that in our scheme the emitted photons propagate forward and backward [Fig. 2(d)], thus they can adequately interact with lasers on both sides of the foil, creating electron-positron pairs on both sides. This is different from the one-laser case [Fig. 2(b)], where the emitted photons just propagate forward and have much less of a chance to further interact with the laser for pair production.

The high-energy $\gamma$-ray photons further interact with laser fields, creating a large number of electron-positron pairs at the antinodes of the formed standing waves. The created pairs are then quickly trapped between electric-field peaks of the standing wave, which are distributed periodically at the electric-field nodes $(E=0)$ with an interval of $0.5 \lambda$, shown in Fig. 1(c) and more clearly in Fig. 1(d), i.e., in the normal radiation trapping (NRT) regime [31]. This is in agreement with the theory of "phase-space contraction" $[42,43]$ because the entropy of the system decreases with time, $d S / d t=\int d^{3} q d^{3} p f \nabla_{p} \cdot \mathbf{F}_{\mathrm{RR}} \leqslant 0$, due to the radiation losses, where $S=-\int d^{3} q d^{3} p f \ln f$ is the entropy associated with a distribution of particles with the distribution function $f=f(\mathbf{q}, \mathbf{p}, t)$ (q and $\mathbf{p}$ are particle position and momentum vectors, respectively), and $\mathbf{F}_{\mathrm{RR}}$ is the Frenkel force, written as $\mathbf{F}_{\mathrm{RR}}=2 e^{2} \omega / 3 m c^{3}$ $\left\{\mathbf{E}(\mathbf{v} \cdot \mathbf{E})+\mathbf{E} \times \mathbf{B}+\mathbf{B} \times(\mathbf{B} \times \mathbf{v})-\gamma \mathbf{v}\left[(\mathbf{E}+\mathbf{v} \times \mathbf{B})^{2}-(\mathbf{E} \cdot \mathbf{v})^{2}\right]\right\}$. Contrary to the case in Ref. [42], in which the standing wave is formed directly by two laser fields with the same amplitude, in our scheme a part of the incoming laser energy is converted to $\gamma$-rays, which causes the amplitude of the reflected laser field to be smaller than the incoming one, leading to only one attractor (with positive $p_{x}$ ) for stochastic heating of electrons and positrons. This helps to collect the trapped pairs into the middle part.

When the hole boring ends, the electrostatic and thermal pressures increase rapidly and their sum exceeds the laser radiation pressure on each side, leading to heavy decompression and expansion of the foil. According to Eq. (2), the temperature of the electrons in the center is estimated to be about $350 \mathrm{MeV}$ [see Fig. 2(e)] with $\gamma \simeq 700$, and the thermal expansion of the foil takes about $9 T_{0}$ until it is transparent. At time $t=18 T_{0}$, when the foil becomes transparent, the laser penetrates through the foil [see Figs. 1(e) and 2(f)], and the standing waves vanish on each side. Afterward, the trapped electrons and positrons are pushed and collected into the center by their radiation pressures from both sides and retrapped at the center due to a new standing wave formed directly by two lasers, as shown in Figs. 1(f) and 1(h). It can be seen
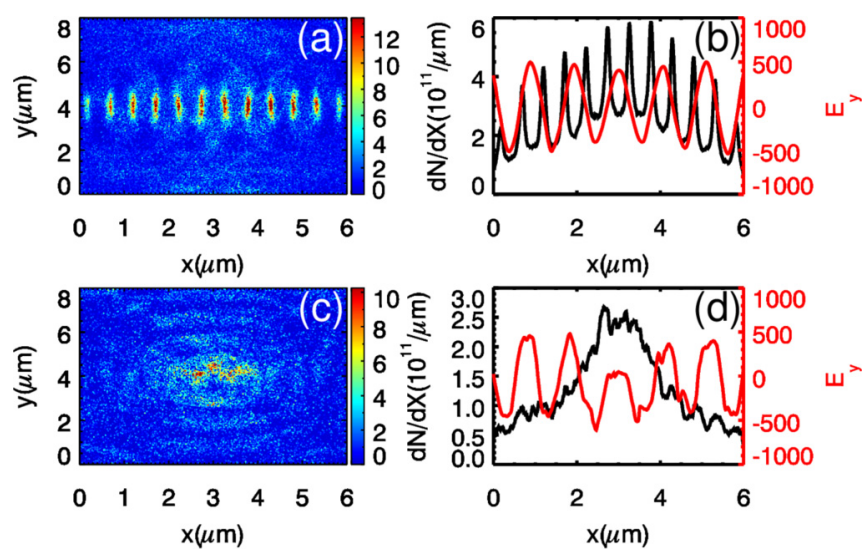

FIG. 3. (Color online) (a) and (c) The density maps of created positrons at $t=18 T_{0}$ when two ultraintense lasers irradiate a solid $\mathrm{Al}$ foil, where the foil thickness is chosen to be $l_{0}=0.5$ (a) and $3.5 \mu \mathrm{m}$ (b), respectively, breaking the conditions (3) and (4). All other parameters are the same as those in Fig. 1. Parts (b) and (d) are their longitudinal distributions (black) as well as the longitudinal profiles of electric fields $E_{y}$ (red), respectively.

that the maximum number density of the pair plasma is about $4.5 \times 10^{22} \mathrm{~cm}^{-3}$, which is two orders of magnitude higher than previously obtained [28-30]. In addition, the energy spectra of the created electrons and positrons [shown in Fig. 2(e)] have similar profiles, in agreement with Refs. [16,17]. At $t=20 T_{0}$ after the interaction, the number of produced $\gamma$-ray photons is about $1.5 \times 10^{14}$ and that of the positrons is $1.2 \times 10^{12}$, which covers, respectively, $22 \%$ and $0.21 \%$ of the total incident laser energy. This positron number is also about two orders of magnitude higher than that obtained in Refs. $[28,39]$ with 10 PW lasers.

As mentioned above, to achieve the enhanced pair production scheme, laser and target conditions need to satisfy Eqs. (3) and (4). If the matching condition is broken, the enhancement will be greatly weakened. Figures 3(a) and 3(b) give the results of the case in which the foil is too thin so that the lasers penetrate the foil too early, where $l_{0}=0.5 \mu \mathrm{m}$ is taken. The results show that due to too early penetration of the laser through the foil, the collective, kinetic laser-solid target interaction has not been adequately developed and the hot electrons act only as seeds in the standing waves formed directly by two incoming lasers, which emit photons. The photons then create pairs, similarly to the conventional cascade pair production $[24,25]$. Because there is no self-collection effect, the pairs distribute (and are trapped) in the nodes of the standing waves with a maximum density of about $1.1 \times 10^{22} \mathrm{~cm}^{-3}$, which is about $1 / 4$ of that in our scheme. Figures $3(\mathrm{c})$ and $3(\mathrm{~d})$ show the results of the other case in which the foil is too thick to be penetrated, where $l_{0}=3.5 \mu \mathrm{m}$ is taken and the other parameters are the same as above. We can see that since the two incident lasers cannot penetrate through the foil, electrons and positrons have a chaotic distribution, leading to a significant drop of the pair density to $9 \times 10^{21} \mathrm{~cm}^{-3}$, much smaller than that in Fig. 1(d).

Lastly, as we know, with the rapid progress in laser technology, laser intensity can be further increased up to $10^{24} \mathrm{~W} / \mathrm{cm}^{2}$ in the future. Figure 4 shows the number of 


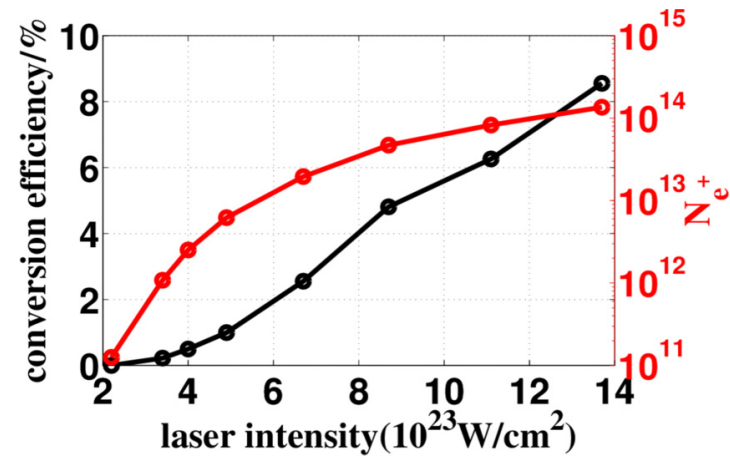

FIG. 4. (Color online) The total number of the created positrons (red) and the conversion efficiency (black) from laser to pairs scaling with the laser intensities in our enhanced scheme, where conditions (3) and (4) are satisfied.

created positrons in total and the conversion efficiency from the laser to the positron pairs with increasing intensities in our scheme with conditions (3) and (4) satisfied. It is clear that both the produced positron number and the conversion efficiency increase significantly with laser intensities. When the intensity rises to $I_{0}=1.4 \times 10^{24} \mathrm{~W} / \mathrm{cm}^{2}$, the number of positrons produced by our enhanced scheme can reach $2.0 \times 10^{14}$ with a laser-to-positron conversion efficiency of about $10 \%$, where the created pair plasma density is as solid as $100 \mathrm{~s}$ of $n_{c}$. This relativistic pair plasma is much denser than those obtained through the general cascade pair production scheme [25] due to the above self-collection effect. However, the total positron number gradually becomes saturated and is comparable with that obtained in the cascade scheme due to the higher rate of cascade development when the laser intensity $I>10^{24} \mathrm{~W} / \mathrm{cm}^{2}$. Also note that the square temporal profile of the laser pulse is taken here to enable a better comparison of the simulation results with the theory [Eqs. (1)-(4)]. Simulation with a laser pulse of a more realistic Gaussian temporal profile (pulse duration $60 \mathrm{fs}$ ) is also carried out, which shows that the maximum positron density of $1.2 \times 10^{22} \mathrm{~cm}^{-3}$ (about $10^{12}$ positrons) can be obtained, which is a bit lower than the density obtained herein. Furthermore, in a more realistic three-dimensional (3D) simulation, due to an additional plasma expansion along the $z$ dimension, the maximum compressed density of the foil target may be lower, and the foil expansion from compression to transparency may become faster, in comparison with the 2D case mentioned herein. Both of these lead to a shorter lifetime of the stable standing waves, which may eventually result in a small reduction of positron pair productions. In addition, the positrons created and trapped by NRT may have a small leak through the additional $z$ axis before self-collection, where the electric field $E_{z}=0$. Therefore, we estimate that the final pair plasma density and the total positron number may become a bit lower in the $3 \mathrm{D}$ case. However, since the laser we use is linearly $p$-polarized along the $y$ direction in the current 2D $x-y$ simulation plane, where the exact QED pair production physics occurs, the dominant dynamics will not change in a $3 \mathrm{D}$ simulation case.

In conclusion, we have proposed a scheme for enhanced production of dense and high-energy electron-positron pair plasmas by using two ultraintense laser pulses to irradiate a thin foil target from opposite sides. Two-dimensional QED-PIC simulations show that electron-positron-pair plasma sources with an unprecedentedly high density of $4.5 \times 10^{22} / \mathrm{cm}^{3}$ (total particle number of $1.2 \times 10^{12}$ ) and a high energy of $100 \mathrm{~s}$ of $\mathrm{MeV}$ are produced by using $10 \mathrm{PW}$ lasers at intensities $3.4 \times 10^{23} \mathrm{~W} / \mathrm{cm}^{2}$. This enhanced pair production scheme in QED plasmas may be tested using high-power lasers such as ELI [20] in the next few years. Note that the reason that the square temporal profile of the laser pulse is taken here is to better compare the simulation results with the theory [Eqs. (1)-(4)]. Simulations with laser pulses of Gaussian temporal profiles have also been done, and they show similar basic physics.

This work is supported by the National Natural Science Foundation of China, Grants No. 11575298, No. 91230205, No. 11575031, and No. 11175026, the National Basic Research 973 Projects No. 2013CBA01500 and No. 2013CB834100, and the National High-Tech 863 Project. B.Q. acknowledges the support from the Thousand Young Talents Program of China. M.B. and M.Z. acknowledge support from the Engineering and Physical Sciences Research Council (EPSRC), Grant No. EP/1029206/1.
[1] P. A. M. Dirac, Proc. R. Soc. London, Ser. A 117, 610 (1928).

[2] D. C. Anderson, Phys. Rev. 43, 491 (1933).

[3] D. L. Burke et al., Phys. Rev. Lett. 79, 1626 (1997).

[4] V. Tsytovich and C. B. Wharton, Comments Plasma Phys. Control. Fusion 4, 91 (1978).

[5] E. P. Liang and C. D. Dermer, Opt. Commun. 65, 419 (1988).

[6] D. B. Cassidy and A. P. Mills Jr., Nature (London) 449, 195 (2007).

[7] J. Wardle et al., Nature (London) 395, 457 (1998).

[8] P. Meszaros, Annu. Rev. Astron. Astrophys. 40, 137 (2002).

[9] R. D. Blandford and R. L. Znajek, Mon. Not. R. Astron. Soc. 179, 433 (1977).
[10] M. C. Begelman, D. Blandford, and J. Rees, Rev. Mod. Phys. 56, 255 (1984).

[11] P. Chang, A. Spitkovsky, and J. Arons, Astrophys. J. 674, 378 (2008).

[12] M. Hoshino et al., Astrophys. J. 390, 454 (1992).

[13] W. Heitler, The Quantum Theory of Radiation (Clarendon, Oxford, 1954).

[14] K. Nakashima and H. Takabe, Phys. Plasmas 9, 1505 (2002).

[15] E. P. Liang, S. C. Wilks, and M. Tabak, Phys. Rev. Lett. 81, 4887 (1998).

[16] H. Chen, S. C. Wilks, J. D. Bonlie, E. P. Liang, J. Myatt, D. F. Price, D. D. Meyerhofer, and P. Beiersdorfer, Phys. Rev. Lett. 102, 105001 (2009). 
[17] H. Chen et al., Phys. Rev. Lett. 105, 015003 (2010).

[18] G. Sarri et al., Nature Commun. 6, 6747 (2014).

[19] V. Yanovsky et al., Opt. Express 16, 2109 (2008).

[20] http://www.eli-laser.eu

[21] J. Schwinger, Phys. Rev. 82, 664 (1951).

[22] A. M. Fedotov, N. B. Narozhny, G. Mourou, and G. Korn, Phys. Rev. Lett. 105, 080402 (2010).

[23] G. Breit and J. A. Wheeler, Phys. Rev. 46, 1087 (1934).

[24] A. R. Bell and J. G. Kirk, Phys. Rev. Lett. 101, 200403 (2008).

[25] E. Nerush, I. Y. Kostyukov, A. M. Fedotov, N. B. Narozhny, N. V. Elkina, and H. Ruhl, Phys. Rev. Lett. 106, 035001 (2011).

[26] J. Kirk et al., Plasma Phys. Control. Fusion 55, 095016 (2013).

[27] S. S. Bulanov, T. Z. Esirkepov, A. G. R. Thomas, J. K. Koga, and S. V. Bulanov, Phys. Rev. Lett. 105, 220407 (2010).

[28] C. P. Ridgers, C. S. Brady, R. Duclous, J. G. Kirk, K. Bennett, T. D. Arber, A. P. L. Robinson, and A. R. Bell, Phys. Rev. Lett. 108, 165006 (2012).

[29] C. P. Ridgers et al., Phys. Plasmas 20, 056701 (2013).

[30] C. S. Brady et al., Phys. Plasmas 21, 033108 (2014).

[31] J. G. Kirk et al., Plasma Phys. Control. Fusion 51, 085008 (2009).
[32] A. Gonoskov, A. Bashinov, I. Gonoskov, C. Harvey, A. Ilderton, A. Kim, M. Marklund, G. Mourou, and A. Sergeev, Phys. Rev. Lett. 113, 014801 (2014).

[33] A. Macchi, F. Cattani, T. V. Liseykina, and F. Cornolti, Phys. Rev. Lett. 94, 165003 (2005).

[34] P. Gibbon, Phys. Rev. E 72, 026411 (2005).

[35] A. P. L. Robinson et al., Plasma Phys. Control. Fusion 51, 024004 (2009).

[36] B. Qiao, M. Zepf, M. Borghesi, and M. Geissler, Phys. Rev. Lett. 102, 145002 (2009).

[37] C. S. Brady and C. P. Ridgers, Plasma Phys. Control. Fusion 55, 124016 (2013).

[38] L. Yin, B. J. Albright, J. K. Bowers, D. Jung, J. C. Fernández, and B. M. Hegelich, Phys. Rev. Lett. 107, 045003 (2011), and references therein.

[39] C. P. Ridgers et al., J. Comput. Phys. 260, 273 (2014).

[40] R. Duclous, J. G. Kirk, and A. R. Bell, Plasma Phys. Control. Fusion 53, 015009 (2011).

[41] H. Chen et al., Phys. Plasmas 16, 122702 (2009).

[42] G. Lehmann and K. H. Spatschek, Phys. Rev. E 85, 056412 (2012).

[43] M. Tamburini et al., Nucl. Instrum. Methods Phys. Res., Sect. A 653, 181 (2011). 